\title{
Persian sentiment analysis of an online store independent of pre-processing using convolutional neural network with fastText embeddings
}

\author{
Sajjad Shumaly ${ }^{1}$, Mohsen Yazdinejad ${ }^{2}$, Yanhui Guo ${ }^{\text {Corresp. } 3}$ \\ ${ }^{1}$ Industrial Engineering, Sharif University of Technology, Tehran, Iran \\ 2 Computer Engineering, Isfahan University, Isfahan, Iran \\ ${ }^{3}$ Computer Science, University of Illinois at Springfield, Springfield, United States \\ Corresponding Author: Yanhui Guo \\ Email address: yguo56@uis.edu
}

Sentiment analysis plays a key role in companies, especially stores, and increasing the accuracy in determining customers' opinions about products assists to maintain their competitive conditions. We intend to analyze the users' opinions on the website of the most immense online store in Iran; Digikala. However, the Persian language is unstructured which makes the pre-processing stage very difficult and it is the main problem of sentiment analysis in Persian. What exacerbates this problem is the lack of available libraries for Persian pre-processing, while most libraries focus on English. To tackle this, approximately 3 million reviews have been gathered in Persian from the Digikala website using web-mining techniques and have been used the fastText method to create a word embedding. It has been assumed it would dramatically cut down the need for the text pre-processing through the skip-gram method considering the position of the words in the sentence and the words' relations to each other. Another word embedding has been created using the TF-IDF in parallel with fastText to compare their performance. In addition, the results of the CNN, BiLSTM, Logistic Regression, and Naïve Bayes models have been compared. As a significant result, we obtained 0.996 AUC, and 0.956 F-score using fastText and CNN. In this article, not only it has been demonstrated to what extent it is possible to be independent of pre-processing but also the accuracy obtained is also better than other researches done in Persian. Avoiding complex text preprocessing is also important for other languages since most text preprocessing algorithms have been developed for English and cannot be used for other languages. The created word embedding due to its high accuracy and independence of pre-processing has other applications in Persian besides sentiment analysis. 
2 Persian Sentiment Analysis of an Online Store

3

4

5

6

7

8

9

10

11

12

13

14

15

16

17

\section{independent of pre-processing using Convolutional Neural Network with fastText Embeddings}

Sajjad Shumaly ${ }^{1}$, Mohsen Yazdinejad², Yanhui Guo ${ }^{3}$

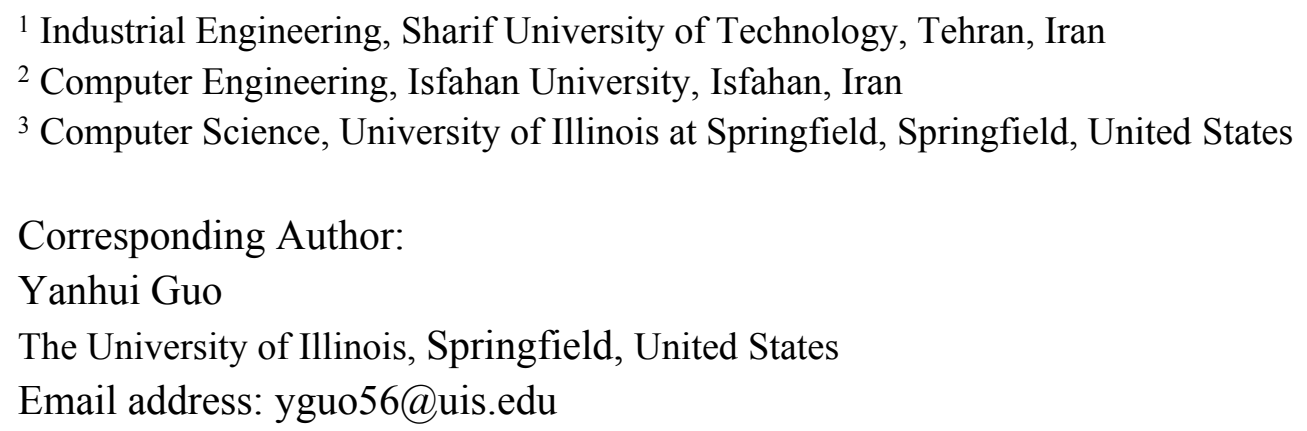

\section{Abstract}

Sentiment analysis plays a key role in companies, especially stores, and increasing the accuracy in determining customers' opinions about products assists to maintain their competitive conditions. We intend to analyze the users' opinions on the website of the most immense online store in Iran; Digikala. However, the Persian language is unstructured which makes the pre-processing stage very difficult and it is the main problem of sentiment analysis in Persian. What exacerbates this problem is the lack of available libraries for Persian pre-processing, while most libraries focus on English. To tackle this, approximately 3 million reviews have been gathered in Persian from the Digikala website using web-mining techniques and have been used the fastText method to create a word embedding. It has been assumed it would dramatically cut down the need for the text preprocessing through the skip-gram method considering the position of the words in the sentence and the words' relations to each other. Another word embedding has been created using the TFIDF in parallel with fastText to compare their performance. In addition, the results of the CNN, BiLSTM, Logistic Regression, and Naïve Bayes models have been compared. As a significant result, we obtained $0.996 \mathrm{AUC}$, and $0.956 \mathrm{~F}$-score using fastText and CNN. In this article, not only it has been demonstrated to what extent it is possible to be independent of pre-processing but also the accuracy obtained is also better than other researches done in Persian. Avoiding complex text preprocessing is also important for other languages since most text preprocessing algorithms have been developed for English and cannot be used for other languages. The created word embedding due to its high accuracy and independence of pre-processing has other applications in Persian besides sentiment analysis. 


\section{Introduction}

With the advancement of technology and the spread of the Internet, proper conditions have been provided for the online store's activities. Due to some advantages such as high variety, delivery speed, and time savings, the customers of this type of store are constantly increasing (Liang and Wang 2019). When buying from online stores, due to the gap between the buyer and the product, there may be some problems such as poor quality of products, inadequate after-sales service, or inconsistency between product descriptions and performance ( $\mathrm{Ji}$, Zhang, and Wang 2019). One of the viable solutions to overcome the problems is to use the opinion of users who have already purchased the product.

In the past, if people needed to know the other's opinion, they would ask questions of family, friends, or relatives. Similarly, companies and stores used surveys to find out the opinions of people or customers. But today, if people require to buy or companies and stores need to know the opinions of customers to provide better services and products, they can easily refer to people's comments and discussions on online store websites or forums. Therefore, online reviews are important sources of information about the quality of goods that play a key role in customer awareness of products (X. Li, Wu, and Mai 2019). Online reviews enable the customer to have a comprehensive view of the products and their alternatives before making a purchase, thus, it has a significant impact on the extent of product sales (Hu, Liu, and Zhang 2008). As a matter of fact, the immediate response of stores to their customers' complaints is essential in maintaining their competitive position. But analyzing these reviews manually is quite time-consuming and costly. Also, automatic comment analysis has some obstacles, problems such as using sentences with incorrect grammar, using slang terms, and not following the correct punctuation are an integral part of making text analysis difficult (Irfan et al. 2015). When it comes to resolving these problems, sentiment analysis techniques play an essential role. These techniques automatically estimate customer sentiment into positive, negative, and even neutral classes. Therefore, sentiment analysis for online stores is highly valued because it can extract users' sense of goods and help to make decisions to increase customer satisfaction and product sales. Sentiment analysis can be considered as a type of content analysis that specifically seeks to determine the emotional tone of the text (Oscar et al. 2017). This is done based on the emotional evidence between words and phrases (Tausczik and Pennebaker 2010).

In this article, we are seeking to analyze the feelings of customer reviews on the website of the largest and well-known online store in Iran (Digikala). At first, lingual problems were taken into account as a significant challenge. There are several problems in Persian text pre-processing such as using slang, using letters of other languages especially Arabic, lack of a clear boundary between phrases. To tackle the problems, we employed fastText and skip-gram because we wanted to examine whether the utilize of the methods capable of reducing the need for data pre-processing and make language processing easier. In the following, we will inspect this assumption and compare the obtained results with other algorithms and other reports. Another severe limitation was that the deep learning models required an immense dataset, but most of the available datasets in Persian are small to such an extent that they cannot be employed in deep models. Thus, a rich 
and immense dataset had to be extracted from the Digikala website which was conducted by webmining methods. It should be noted that this article seeks to achieve the following goals:

- Investigating the reduction of the need for text pre-processing by implementing methods such as fastText and skip-gram, either in Persian language processing or others.

- Gathering comprehensive customer reviews dataset based on various types of digital goods to create a general word embedding for a various range of works related to digital goods.

- Sentiment analysis of Digikala website's reviews with high accuracy even compared to other researches.

\section{Related Works}

Sentiment analysis methods are divided into three general categories Lexicon based, traditional Machine Learning, and Deep Learning models (Yadav and Vishwakarma 2020). The first category is the sentiment analysis using a sentiment lexicon and it is an unsupervised method. In this case, emotional similarities of words and phrases are used and its accuracy is highly dependent on prelearned weights (Taboada et al. 2011). This method collects a set of pre-compiled sentiment words, terms, phrases, and idioms with a specific thematic category such as opinion finder lexicon (Wilson et al. 2005) and ontologies (Kontopoulos et al. 2013).

The second category is based on machine learning methods which are divided into supervised and unsupervised categories. The accuracy of these methods is strongly influenced by the extracted features from the text. Supervised techniques such as Naïve Bayes, SVM, Maximum Entropy, and Logistic Regression are the most common techniques in this field (Ye, Zhang, and Law 2009)(Montejo-Ráez et al. 2014). However, unsupervised methods are suitable for situations where labeling for the dataset is impossible (Paltoglou and Thelwall 2012).

Deep learning has grown and been used in many areas in the last decade, for example in the field of object recognition (Ghoreyshi, AkhavanPour, and Bossaghzadeh 2020)(Ali et al. 2020), speech recognition (Deng, Hinton, and Kingsbury 2013)(H. Li, Baucom, and Georgiou 2020), anomaly detection (Zhao et al. 2018), feature extraction (Lin, Nie, and Ma 2017)(Rajaraman et al. 2018), auto-encoding ( $\mathrm{Pu}$ et al. 2016). Also, in cases where deep learning along with machine learning has been used for text analysis and sentiment analysis, good results have been obtained (Tang, Qin, and Liu 2015)(Severyn and Moschitti 2015). The main difference between sentiment analysis by deep learning and other methods is how to extract the features. To be specific, one of the advantages of deep learning models is that there is no need for user intervention in feature extraction, which of course requires a large amount of data to perform the feature extraction operation. Recurrent Neural Network (RNN), Convolutional Neural Network (CNN), Long ShortTerm Memory (LSTM), and Gated Recurrent Unit (GRU) are the most common models of deep learning in sentiment analysis (Zhang, Wang, and Liu 2018).

The most basic and widely used CNN model for sentiment analysis at the sentence level is the one presented by Kim (Kim 2014). Then, Zhang and Wallace proposed a special single-layer CNN architecture by examining improvements made by changing the model configuration (Zhang and Wallace 2015). Many developments have been made to improve the performance of CNN-based 
119

120

121

122

123

124

125

126

127

128

129

130

131

132

133

134

135

136

137

138

\section{9}

140

141

142

143

144

145

146

147

148

149

150

151

152

153

154

155

156

sentiment analysis models. In this regard, an example of CNN combined with fuzzy logic called the Fuzzy Convolutional Neural Network (FCNN) (Nguyen, Kavuri, and Lee 2018) is noteworthy. The use of CNN in natural language processing is now a common topic and much research is being done in this area (Wehrmann et al. 2017)(Gan et al. 2020)(Arora and Kansal 2019).

Deep neural networks are difficult to train because they often suffer from the problem of vanishing gradients. LSTM architecture was introduced (Hochreiter and Schmidhuber 1997) to overcome this shortcoming to learn long-term dependencies. After the original work, the LSTM has experienced several improvements such as adding forget gate (Gers 1999). A neural network architecture could not be so great adopted into practice without strong theoretical support, therefore, a widespread review considering the several LSTM variants and their performances relative to the so-called vanilla LSTM model was conducted by Greff et al. (Greff et al. 2017). The vanilla LSTM model is interpreted as the primary LSTM block with the addition of the forget-gate and peephole connections. Also, to overcome some limitations in conventional RNN models, bidirectional RNN (BRNN) models were proposed. Using this model's structure, both future and past situations of sequential inputs in a time frame are evaluated without delay (Schuster and Paliwal 1997). By combining the ideas of BRNN and LSTM it is possible to achieve Bidirectional LSTM (BiLSTM) which has better performance than LSTM in classification processes. With the development of LSTM in recent years, it has been used in projects such as Google Translate and Amazon Alexa (Wu et al. 2016)(Vogels 2016) in natural language processing.

\section{Materials and methods}

All the taken steps, methods, codes, and results that are presented below, along with a part of the extracted dataset are fully accessible on the repository (Yazdinejad and Shumaly 2020).

\subsection{Dataset}

Having access to a large dataset with richness and content integrity is indispensable to train a deep model. Most available datasets to train a deep model and sentiment analysis are in English. To collect a rich dataset, web-mining methods were used and the reviews on the Digikala website were extracted which were in Persian. Posted reviews by buyers express their level of satisfaction with their purchase and product features. After submitting their reviews, buyers could choose between the "I suggest" and "I do not suggest" options. These two options were extracted and used in the model as labels for the problem of sentiment analysis. Our goal was to analyze the opinions of users of the Digikala website, so we extracted the data of the section related to digital goods using web-mining libraries such as the Beautiful Soup (Richardson 2020). Beautiful Soup is a Python package to parse XML and HTML documents and it is useful for web scraping (Hajba 2018).

\subsection{Pre-processing}


157 One of the first steps in natural language processing problems has always been data pre-processing.

158

159

160

161

162

163

164

165

166

167

168

169

170

171

172

173

174

175

176

177

178

179

180

181

182

183

184

185

186

187

188

189

190

191

192

193

194

At this stage, the texts need to be cleaned and prepared to begin the analysis. In Persian, this stage is even more difficult and important because it has its complexities. This field has attracted many researchers in the last decade, therefore, libraries and algorithms in the field of pre-processing in Persian have been developed (Mohtaj et al. 2018)(Nourian 2013) which have become more complete and better over time. However, these algorithms cannot work as well as similar algorithms in English and need further development. We are seeking a way to achieve an accurate result by avoiding the complications of data pre-processing steps in Persian. Regular expressions are used for data pre-processing in all of the following steps using the "re" library (Rachum 2020) in python. Pre-developed libraries for the Persian language have not been used to perform data pre-processing steps and we assume that the use of fastText and skip-gram in creating word embedding reduces the need for complex pre-processing.

\subsubsection{Normalization}

In Persian, some letters are not unique and may have different alternatives to other languages such as Arabic. For example, the letter "ى "ي" is Persian, but the letter Arabic, and these two letters will likely be used interchangeably. This causes the created words to be considered as two different words. In this way, they may be considered separately in the calculations and a vector can be drawn for each with its characteristics. To solve this issue, it is necessary to use the standard form for all available texts.

\subsubsection{Tokenization}

Tokenization is a stage in which attempts are made to divide sentences into meaningful words and phrases that can be considered as a suitable input for the next steps. The main challenge of the Persian language at this stage is that sometimes there are no clear boundaries between phrases as a result of three different modes of spacing in Persian. In other words, phrases in Persian can be without space, with half-space, or with space, which is often mistakenly used instead of each other. For instance, the words "نرمافزار" and افزار", which both mean software, are written with both space and half-space forms. If the wrong form is used, the phrase border will be mistakenly recognized as two separate words "نرم" and "فزار". Vice versa, phrases that consist of several words

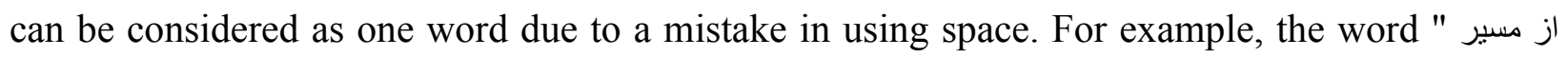
ديكر", which means "from another path", maybe written as "ازمسيرديكر" without any spaces. These kinds of mistakes blur the line between phrases and words and make it difficult to pre-process.

\subsubsection{Stemming}

The stemming process seeks to remove part of the word in such a way that the root of the word is determined (Willett 2006). The root of the word does not necessarily mean the dictionary root of the word and is acceptable in cases where it can improve the performance of the model. For

PeerJ Comput. Sci. reviewing PDF | (CS-2020:10:54625:2:0:NEW 5 Feb 2021) 
195

196

197

198

199

200

201

202

203

204

205

206

207

208

209

210

211

212

213

214

215

216

217

218

219

220

221

222

223

224

225

226

227

228

229

230

example, we can refer to the phrase "رنگ هايشان". In this phrase, "رنگ means color, and "هان" is used to represent plural and "يثان" for determination of ownership. A significant number of stemming algorithms use the following rule (Mohtaj et al. 2018):

(possessive suffix)(plural suffix)(other suffixes)(stem)(prefixes)

Stemming is a rule-based process that is usually done by removing suffixes and prefixes. Consequently, it cannot be used in other languages and each language requires its algorithms. Stemming algorithms are being developed in Persian but due to the complexity of the Persian language, their performance needs to be improved.

\subsection{Pseudo labeling}

In classification problems, it is a common problem that a large number of samples do not have labels and therefore cannot be used in model training. Techniques such as pseudo-labeling can be used to overcome this problem and determine the labels of some samples (Lee 2013). The first step in pseudo-labeling is to develop a model based on labeled samples in the dataset that is in charge of determining the label of unlabeled samples. Only labels that have been estimated with high confidence are accepted. In the next step, another model is developed using training data along with the new labeled data which can be used to predict test data with higher accuracy. In this way, 104.8 thousand Negative Feedback reviews and 30.5 thousand Positive Feedback reviews were labeled and could be used in the dataset for subsequent analysis. As will be shown in the results section, this method had a significant impact on improving accuracy.

\subsection{Data balancing}

Unequal distribution of data in different classes in a classification problem leads to data imbalance. The class with the most data is called the majority class, and the class with the least data is called the minority class. In these cases, the models tend to ignore the minority class and predict in favor of the majority class. Many machine learning models, such as Support Vector Machine, Naïve Bayes, Decision Tree, and Artificial Neural Network cannot have good results in this situation (Díez-Pastor et al. 2015)(Vorraboot et al. 2015). In general, data balancing solutions can be divided into two categories; over-sampling and under-sampling. The goal of both solutions is to approximate the number of data distributed in the minority and majority classes. In over-sampling, this is done by increasing the amount of data in the minority class, and in under-sampling by reducing the amount of data in the majority class. In the present problem, we used the random oversampling method to balance the dataset.

\subsection{Feature Extraction}

\subsection{1. fastText}


231 Neural network-based methods have become very popular in the field of natural language

232

233

234

235

236

237

238

239

240

241

242

243

244

245

246

247

248

249

250

251

252

253

254

255

256

257

258

259

260

261

262

263

264

265

266

267

268 processing due to their accuracy. However, most of these methods are slow to analyze large datasets and they need to receive word embedding to analyze texts. For this reason, a method called fastText has been proposed (Joulin et al. 2016). fastText is an efficient, fast, and open-source model that Facebook has recently released. In fastText, a set of tricks has been used to improve the processing speed and performance of the model, one of which is skip-gram. Data sparsity has always been one of the biggest problems in natural language analysis. In other words, the main problem of modern language processing is that language is a system of rare events, so varied and complex, that we can never model all possibilities (Preethi Krishna and Sharada 2020). Therefore, skip-gram allows some words to be skipped and non-adjacent words to be examined together. Mikolov et al (Mikolov et al. 2013) found the skip-gram model to be superior to the bag-of-word model in a semantic-syntactic analogy task. Skip-gram is popular, easy to implement, and it is proven and reliable (Gurunath Shivakumar and Georgiou 2019). Accordingly, in this article, word embeddings have been provided using fastText and skip-gram to investigate the reduction of language processing dependence on data-preprocessing.

\subsection{Sentiment analysis model 3.6.1. Convolution neural network}

Using CNN has shown high accurate results based on studies in English texts (Nedjah, Santos, and de Macedo Mourelle 2019). This model can receive and analyze word embedding as input instead of images, which are also effective in this area (Kim 2014). Each row of the input matrix represents a word. Figure 1 shows the architecture of a CNN model used for the NLP classification problem (Zhang and Wallace 2015). This figure shows how a CNN model treats a 6-word sentence. The matrix formed for this sentence is analyzed by 6 different convolution filters and converted to maps of attributes with dimensions of $1 \times 4,1 \times 5$, and $1 \times 6$. Finally, the pooling operation is performed on the maps and their outputs are merged to create a unique vector that can be used as input for the SoftMax layer and determine the class. The CNN model used in this article is based on the mentioned model and its architecture is shown in table 1.

\subsubsection{Bidirectional Long Short-Term Memory (BiLSTM)}

Another deep model used to solve the problem is BiLSTM. The LSTM model can decide which information is useful and should be preserved and which information can be deleted based on the dataset it has trained with. The LSTM has been widely used in NLP such as long document categorization and sentiment analysis (Rao et al. 2018). Figure 2 is a demonstration of an LSTM cell used in this article, which has an input layer, an output layer and a forget layer (Gers 1999). Based on the figure, the LSTM cell mathematically expressed as follows:

$$
\begin{aligned}
& f_{t}=\sigma\left(W_{f h} h_{t-1}+W_{f x} x_{t}+b_{f}\right) \\
& i_{t}=\sigma\left(W_{i h} h_{t-1}+W_{i x} x_{t}+b_{i}\right)
\end{aligned}
$$


269

270

271

272

273

274

275

276

277

278

279

280

281

282

283

284

285

286

287

288

289

290

291

292

293

294

295

296

297

298

299

300

301

302

303

304

305

$$
\tilde{c}_{t}=\tanh \left(W_{\tilde{c} h} h_{t-1}+W_{\tilde{c} x} x_{t}+b_{\tilde{c}}\right)
$$

where $x_{t}$ denotes the input; $h_{t-1}$, and $h_{t}$ denote the output of the last LSTM unit and current output; $c_{t-1}$, and $c_{t}$ denote memory from the last LSTM unit and cell state; $f_{t}$ denotes forget gate value; $W_{i}, W_{\tilde{c}}$, and $W_{o}$ are the weights; $b$ is the bias; the operator '.' denotes the pointwise multiplication of two vectors. In LSTM, the input gate can decide what new information can be stored in the cell state, also the output gate can decide what information can be output based on the cell state. By combining the ideas of BRNN and LSTM it is possible to achieve Bidirectional LSTM (BiLSTM) which has better performance than LSTM in classification processes especially in speech processing tasks (Graves and Schmidhuber 2005). Therefore, this article uses the BiLSTM structure, and figure 3 is shown a basic structure of the BiLSTM network (Yildirim 2018). The BiLSTM model used in this article architecture is shown in table 2.

\subsection{Evaluation}

Due to imbalanced data, indicators such as accuracy is not appropriate for this study. Because the developed model in the face of this type of data tends to ignore the minority class and can still be accurate. For this purpose, AUC and F-score indexes will be used, which are good choices for problems dealing with imbalanced data (Sokolova, Japkowicz, and Szpakowicz 2006). AUC indicates the area below the diagram in the ROC curve, and the ROC curve is a method for judging the performance of a two-class classifier (Luo et al. 2020). In the ROC curve, the vertical axis is the TPR (represents the true positive rate), Also, the horizontal axis is FPR (represents the false positive rate).

$$
\begin{aligned}
& F P R=\frac{f p}{t n+f p} \\
& T P R=\frac{f p}{t p+f n}
\end{aligned}
$$

- TP: Positive samples are classified as positive

- FN: Positive samples are classified as negative

- TN: Negative samples are classified as negative

- FP: Negative samples are classified as positive

The F-score is the harmonic mean of precision and recall (Velupillai et al. 2009) and represents a weighted average of precision and recall (Gacesa, Barlow, and Long 2016). This index has wide applications in natural language processing (Derczynski 2016), and like the AUC, it can be used in problems involved with imbalanced data.

$$
\text { Precision }=\frac{t p}{t p+f p}
$$


306

307

308

309

310

311

312

313

314

315

316

317

318

319

320

321

322

323

324

325

326

327

328

329

330

331

332

333

334

335

336

337

338

339

340

341
Recall $=\frac{t p}{t p+f n}$

$F-$ score $=2 \times \frac{\text { Precision } \times \text { Recall }}{\text { Precision }+ \text { Recall }}$

All the steps mentioned in the methodology section can be summarized in figure 4.

\section{Results and discussion}

4.1. Dataset

The digital goods' reviews of the Digikala website were extracted, which are a total of 2,932,747 reviews. Figure 5 shows the frequency of comments per category. Examining the comments of different product categories can increase the comprehensiveness of the model. To be specific, the words, phrases, and sentences are different in reviews of the products in the different categories, and considering various types of product categories will improve the generalization of the model in different situations. Table 3 shows the general structure of the collected dataset. In this table, the "Comment ID" column stores the unique values for each comment, the "Original Comment" column is the original of the comments written in Persian, the "Translated Comment" column is a translation of the "Original Comment" column into English. The "Translated Comment" column is used only to increase readability in the table and does not exist in the dataset. In the "Negative Feedback" column, if the value is 1 , means that the user is not advised to buy the product, and in the "Positive Feedback" column, if the value is 1, it means the user is advised to buy the product, and the "Cat. Name" column represents the product category for which the comment was written.

The positive point of this website is that the buyers after submitting their comments can choose an option that states whether they generally recommend the product to others or not. Therefore, a significant number of extracted reviews are labeled. In other words, 308,122 of the reviews in the dataset do not recommend purchased items to others and the "Negative Feedback" column of these reviews in the dataset shows the number one. Likewise, 1,749,055 of the reviews in the dataset recommend the purchased items to others, and the "Positive Feedback" column of these comments in the dataset shows the number one. A significant part of the reviews is without labels and the reviews with labels are also imbalanced and these problems must be addressed in some ways.

\subsection{Pre-processing}

During the initial review of the comments, the first correction that had to be made was the removal of escape sequences. An escape sequence is a series of two or more characters starting with a backslash and when used in a string, are not displayed as they are written. In most reviews, there were some escape sequences such as " $\backslash \mathrm{n}$ " and " $\mathrm{t}$ " that needed to be removed. Also, sometimes users wrote some URLs to link to personal content that had to be removed. At this stage, all Persian numbers were converted to English, and letters that had different alternatives were standardized to

Peer] Comput. Sci. reviewing PDF | (CS-2020:10:54625:2:0:NEW 5 Feb 2021) 
342

343

344

345

346

347

348

349

350

351

352

353

354

355

356

357

358

359

360

361

362

363

364

365

366

367

368

369

370

371

372

373

374

375

376

377

378

379

380

normalize the text. Then all the phrases were tokenized by defining the word boundary and converting the half-space to space. In the stemming stage, prefixes and suffixes used were removed.

After the pre-processing steps, the number of words in the Positive Feedback class was 6.1 million and the number of words in the Negative Feedback class was 34.1 million. Using the word cloud diagram, the most repetitive words in each of the classes can be depicted. Figure 6 and figure 7 show the repetitive words in the Positive Feedback and Negative Feedback classes, respectively. Words like "I gave back", "bad" and "I do not recommend" can be seen in the Negative Feedback figure, and words like "I'm satisfied", "Appropriate" and "Speed" can be seen in the Positive Feedback figure.

\subsection{Sentiment analysis}

Data balancing is a crucial step that can increase accuracy. The random over-sampling method was used to balance the data. In other words, some data with the label of "Negative Feedback" were randomly selected and repeated. As a matter of fact, one of the common mistakes in this section is to apply the balancing method to the entire data which leads to errors in estimating the indicators. In these cases, the indicators are in a better position than the model capability and the results are reported incorrectly well. To avoid this, the balancing method was used only for the training data. After using the pseudo-labeling method, the number of positive feedbacks was about 1.8 million and the number of negative feedbacks was about 400 thousand. In this way, the negative feedbacks were repeated randomly about four times to balance the dataset.

The stratified K-fold cross-validation method is used to perform the evaluation. It is a method for model evaluation that determines how independent the results of statistical analysis on a dataset are from training data. In K-fold cross-validation, the dataset is subdivided into a $\mathrm{K}$ subset and each time one subset is used for validation and the other $\mathrm{K}-1$ is used for training. This procedure is repeated $\mathrm{K}$ times and all data is used exactly once for validation. The average result of this $\mathrm{K}$ computing is selected as a final estimate. Stratified sampling is the process of dividing members of a dataset into similar subsets before sampling and this type of data sampling was selected due to imbalanced data. Using the stratified K-fold cross-validation method, we expect the values of the indicators to be more real. In all stages of measuring the accuracy of the model, $\mathrm{K}$ was considered equal to 5 .

As stated in the methodology, TF-IDF and fastText methods were used to extract the features. The BiLSTM and CNN models used the fastText output, and the Naïve Bayes and Logistics Regression models used the TF-IDF output, and their accuracy was finally compared with each other in table 4. According to this table, the results of BiLSTM and CNN models are more accurate than others and $\mathrm{CNN}$ has given the best results.

As expected, due to the use of fastText and skip-gram methods, the need for data pre-processing has been reduced. In other words, stemming and normalization methods have not affected the final result. To examine this more closely, we chose the CNN model as the best model and we once 
381

382

383

384

385

386

387

388

389

390

391

392

393

394

395

396

397

398

399

400

401

402

403

404

405

406

407

408

409

410

411

412

413

414

415

416

417

418

419

420

performed the sentiment analysis process using the pre-processing steps and again without these steps. The AUC and F-score were 0.9943 and 0.9291 before pre-processing, and 0.9944 and 0.9288 after pre-processing. The results can be seen in table 5. In the table, the meaning of the "before preprocessing" is just before the stemming and normalization steps. In other words, the methods used to create word embedding can depict the same words in the same range of spaces without the need to standardize letters and also without the need to identify the original root of words.

To implement pseudo-labeling, we developed a model that can estimate labels for unlabeled reviews using fastText and CNN models. After estimating all the labels, those with more than $90 \%$ probability for the Negative Feedback class and less than $1 \times 10^{-7}$ for the Positive Feedback class were selected. Therefore, 104.8 thousand Negative Feedback reviews and 30.5 thousand Positive Feedback reviews were labeled and could be used in the dataset for subsequent analysis. In using the pseudo-labeling technique, most of our focus was on Negative Feedback as a minority class, which also leads to balance the dataset as much as possible. In this way, a significant amount of unlabeled data that had been excluded from the sentiment analysis process was re-entered into the process and helped to increase the accuracy and generalizability of the model.

Contrariwise of pre-processing, the use of the pseudo-labeling method significantly improved the results. After using pseudo-labeling, the values of AUC and F-score improved to 0.996 and 0.956. The values of the three mentioned states can be seen based on different folds in table 5 . Figure 8 also shows the ROC curve for all three states.

The suggested model has had better results than the previous models which have used preprocessing methods in Persian sentiment analysis. For instance, some researchers introduced preprocessing algorithms and succeed to enhance the results of machine learning algorithms (Saraee and Bagheri 2013). In the research, the F-score of the proposed pre-processing algorithms employing Naïve Bayes as a classifier algorithm is 0.878 . In another research, the various alternatives for pre-processing and classifier algorithms were examined and the best result was assisted with an SVM classifier by 0.915 F-score value (Asgarian, Kahani, and Sharifi 2018). Also, some researches were attempted to utilize state-of-the-art deep models in such a way to reduce dependency on pre-processing and avoiding complex steps (Roshanfekr, Khadivi, and Rahmati 2017). The F-score of the BiLSTM and CNN algorithms in the research is 0.532 and 0.534 . All mentioned article's focus was on the digital goods reviews in Persian two-class sentiment analysis as same as this article. A comparison of the results in this paper with other researches and other common algorithms indicates that not only the dependence on data pre-processing has been eliminated but also the accuracy has increased significantly.

The result reveals that it is quite possible to create independent models from the pre-processing process using the method of fastText and skip-gram. Moreover, BiLSTM and CNN methods can have significant results. However, all of the mentioned methods need to have an immense dataset. To prove this, It is noteworthy that the use of the pseudo-labeling method because of increasing training data has a great impact on the result. Independency from pre-processing steps is not related to the Persian language. The results are reachable for other languages using sufficient labeled samples and the mentioned methods in this article. 
422

423

424

425

426

427

428

429

430

431

432

433

434

435

436

437

438

439

440

441

442

443

444

445

446

447

448

449

450

451

452

453

454

455

456

457

458

459

460

\section{Conclusion}

The dataset included approximately 3 million reviews was extracted from the digital goods section of the Digikala website as the largest online store in Iran. Basic pre-processing methods were used to modify the words and tokenize them. Due to the lack of labels for a large part of the dataset, the pseudo-labeling method was employed which improved the results. Data balancing was also performed using random over-sampling. Persian data pre-processing was found difficult, so the fastText method was conducted to reduce the need for data pre-processing and word embedding development. The embeddings were employed as the input to the BiLSTM, and CNN models. Using the suggested model, not only the obtained results have been very desirable and are much more accurate in Persian compared to other reports but also there are no complications related to data pre-processing. The effect of stemming and normalization on the output was evaluated and revealed that the proposed method is not dependent on data pre-processing. Eventually, Besides the comparison of machine learning and deep learning methods in sentiment analysis, the TF-IDF and fastText methods were compared to create word embedding. The best result was associated with fastText and CNN. The main achievement of this model is the reduction of the need for data pre-processing. Data pre-processing in English is convenient and accurate due to the expanded text pre-processing libraries. However, in other languages, data pre-processing is very complicated because of the lack of proper libraries. Over the suggested model was proved that this need is largely solvable $(\mathrm{AUC}=0.996)$ and the pre-processing steps can be reduced to preliminary tokenization processes. Avoiding complex text preprocessing is also important for other languages Since most text preprocessing algorithms have been developed for English and cannot be used for other languages. In other words, the taken steps are possible to be implemented for other languages to achieve the same results independently of pre-processing steps. Moreover, the created word embedding due to its high accuracy can be used in other text analysis problems especially related to online digital goods.

\section{References}

Ali, Abder-Rahman, Jingpeng Li, Guang Yang, and Sally Jane O’Shea. 2020. “A Machine Learning Approach to Automatic Detection of Irregularity in Skin Lesion Border Using Dermoscopic Images." PeerJ Computer Science 6 (June): e268. https://doi.org/10.7717/peerj-cs.268.

Amiri, Fatemeh, Simon Scerri, and Mohammadhassan Khodashahi. 2015. "Lexicon-Based Sentiment Analysis for Persian Text." In Proceedings of the International Conference Recent Advances in Natural Language Processing, 9-16. https://doi.org/10.13140/RG.2.1.2537.8327.

Boiy, Erik, and Marie-Francine Moens. 2009. "A Machine Learning Approach to Sentiment Analysis in Multilingual Web Texts." Information Retrieval 12 (5): 526-58. https://doi.org/10.1007/s10791008-9070-z.

Dashtipour, Kia, Mandar Gogate, Jingpeng Li, Fengling Jiang, Bin Kong, and Amir Hussain. 2020. “A Hybrid Persian Sentiment Analysis Framework: Integrating Dependency Grammar Based Rules and

Peer) Comput. Sci. reviewing PDF | (CS-2020:10:54625:2:0:NEW 5 Feb 2021) 
461

462

463

464

465

466

467

468

469

470

471

472

473

474

475

476

477

478

479

480

481

482

483

484

485

486

487

488

489

490

491

492

493

494

495

496

497

498

499

500

501

Deep Neural Networks." Neurocomputing 380 (March): 1-10.

https://doi.org/10.1016/j.neucom.2019.10.009.

Deng, Li, Geoffrey Hinton, and Brian Kingsbury. 2013. "New Types of Deep Neural Network Learning for Speech Recognition and Related Applications: An Overview." In 2013 IEEE International Conference on Acoustics, Speech and Signal Processing, 8599-8603. IEEE. https://doi.org/10.1109/ICASSP.2013.6639344.

Derczynski, Leon. 2016. "Complementarity, F-Score, and NLP Evaluation." In Proceedings of the Tenth International Conference on Language Resources and Evaluation (LREC'16), 261-66. https://www.aclweb.org/anthology/L16-1040.

Díez-Pastor, José F, Juan J Rodríguez, César García-Osorio, and Ludmila I Kuncheva. 2015. "Random Balance: Ensembles of Variable Priors Classifiers for Imbalanced Data." Knowledge-Based Systems 85 (September): 96-111. https://doi.org/10.1016/j.knosys.2015.04.022.

Forootan, Faezeh, and Mohammad Rabiei. 2019. "Sentiment Analysis User Comments On E-Commerce Online Sale Websites." International Journal of Web Research 2 (2): 1-8. https://doi.org/10.22133/IJWR.2020.210555.1048.

Gacesa, Ranko, David J Barlow, and Paul F Long. 2016. "Machine Learning Can Differentiate Venom Toxins from Other Proteins Having Non-Toxic Physiological Functions." PeerJ Computer Science 2 (October): e90. https://doi.org/10.7717/peerj-cs.90.

Ghoreyshi, Amir Mohammad, Alireza AkhavanPour, and Alireza Bossaghzadeh. 2020. "Simultaneous Vehicle Detection and Classification Model Based on Deep YOLO Networks." In 2020 International Conference on Machine Vision and Image Processing (MVIP), 1-6. IEEE. https://doi.org/10.1109/MVIP49855.2020.9116922.

Gurunath Shivakumar, Prashanth, and Panayiotis Georgiou. 2019. "Confusion2Vec: Towards Enriching Vector Space Word Representations with Representational Ambiguities." PeerJ Computer Science 5 (June): e195. https://doi.org/10.7717/peerj-cs.195.

Hajba, Gábor László. 2018. Website Scraping with Python. Berkeley, CA: Apress. https://doi.org/10.1007/978-1-4842-3925-4.

Hu, Nan, Ling Liu, and Jie Jennifer Zhang. 2008. "Do Online Reviews Affect Product Sales? The Role of Reviewer Characteristics and Temporal Effects." Information Technology and Management 9 (3): 201-14. https://doi.org/10.1007/s10799-008-0041-2.

Irfan, Rizwana, Christine K King, Daniel Grages, Sam Ewen, Samee U Khan, Sajjad A Madani, Joanna Kolodziej, Lizhe Wang, Dan Chen, Ammar Rayes, Nikolaos Tziritas, Cheng-Zhong Xu, Albert Y. Zomaya, Ahmed Saeed Alzahrani and Hongxiang Li. 2015. "A Survey on Text Mining in Social Networks." The Knowledge Engineering Review 30 (2): 157-70. https://doi.org/10.1017/S0269888914000277.

Ji, Pu, Hong-Yu Zhang, and Jian-Qiang Wang. 2019. "A Fuzzy Decision Support Model With Sentiment Analysis for Items Comparison in E-Commerce: The Case Study of Http://PConline.Com." IEEE Transactions on Systems, Man, and Cybernetics: Systems 49 (10): 1993-2004. https://doi.org/10.1109/TSMC.2018.2875163.

Joulin, Armand, Edouard Grave, Piotr Bojanowski, and Tomas Mikolov. 2016. "Bag of Tricks for Efficient Text Classification." ArXiv Preprint ArXiv:1607.01759, July. http://arxiv.org/abs/1607.01759.

Peer] Comput. Sci. reviewing PDF | (CS-2020:10:54625:2:0:NEW 5 Feb 2021) 
502

503

504

505

506

507

508

509

510

511

512

513

514

515

516

517

518

519

520

521

522

523

524

525

526

527

528

529

530

531

532

533

534

535

536

537

538

539

540

541

Kim, Yoon. 2014. "Convolutional Neural Networks for Sentence Classification." ArXiv Preprint ArXiv:1408.5882, August. http://arxiv.org/abs/1408.5882.

Lee, Dong-Hyun. 2013. "Pseudo-Label: The Simple and Efficient Semi-Supervised Learning Method for Deep Neural Networks." Workshop on Challenges in Representation Learning, ICML 3 (2).

Li, Haoqi, Brian Baucom, and Panayiotis Georgiou. 2020. "Linking Emotions to Behaviors through Deep Transfer Learning." PeerJ Computer Science 6 (January): e246. https://doi.org/10.7717/peerjcs.246.

Li, Xiaolin, Chaojiang Wu, and Feng Mai. 2019. "The Effect of Online Reviews on Product Sales: A Joint Sentiment-Topic Analysis." Information \& Management 56 (2): 172-84. https://doi.org/10.1016/j.im.2018.04.007.

Liang, Ruxia, and Jian-qiang Wang. 2019. "A Linguistic Intuitionistic Cloud Decision Support Model with Sentiment Analysis for Product Selection in E-Commerce." International Journal of Fuzzy Systems 21 (3): 963-77. https://doi.org/10.1007/s40815-019-00606-0.

Lin, Yi-zhou, Zhen-hua Nie, and Hong-wei Ma. 2017. "Structural Damage Detection with Automatic Feature-Extraction through Deep Learning." Computer-Aided Civil and Infrastructure Engineering 32 (12): 1025-46. https://doi.org/10.1111/mice.12313.

Luo, Jun, Senchun Chai, Baihai Zhang, Yuanqing Xia, Jianlei Gao, and Guoqiang Zeng. 2020. “A Novel Intrusion Detection Method Based on Threshold Modification Using Receiver Operating Characteristic Curve." Concurrency and Computation: Practice and Experience 32 (14): e5690. https://doi.org/10.1002/cpe.5690.

Mikolov, Tomas, Kai Chen, Greg Corrado, and Jeffrey Dean. 2013. "Efficient Estimation of Word Representations in Vector Space." ArXiv Preprint ArXiv:1301.3781, January. http://arxiv.org/abs/1301.3781.

Mohtaj, Salar, Behnam Roshanfekr, Atefeh Zafarian, and Habibollah Asghari. 2018. "Parsivar: A Language Processing Toolkit for Persian." In Proceedings of the Eleventh International Conference on Language Resources and Evaluation (LREC 2018). https://www.aclweb.org/anthology/L18-1179.

Nedjah, Nadia, Igor Santos, and Luiza de Macedo Mourelle. 2019. "Sentiment Analysis Using Convolutional Neural Network via Word Embeddings." Evolutionary Intelligence, April, 1-25. https://doi.org/10.1007/s12065-019-00227-4.

Nourian, Alireza. 2013. "Hazm: Python Library for Digesting Persian Text." 2013. https://github.com/sobhe/hazm.

Oscar, Nels, Pamela A Fox, Racheal Croucher, Riana Wernick, Jessica Keune, and Karen Hooker. 2017. "Machine Learning, Sentiment Analysis, and Tweets: An Examination of Alzheimer's Disease Stigma on Twitter." The Journals of Gerontology: Series B 72 (5): 742-51. https://doi.org/10.1093/geronb/gbx014.

Preethi Krishna, P., and A. Sharada. 2020. "Word Embeddings - Skip Gram Model." In ICICCT 2019 System Reliability, Quality Control, Safety, Maintenance and Management, 133-39. Singapore: Springer Singapore. https://doi.org/10.1007/978-981-13-8461-5_15.

$\mathrm{Pu}$, Yunchen, Zhe Gan, Ricardo Henao, Xin Yuan, Chunyuan Li, Andrew Stevens, and Lawrence Carin. 2016. "Variational Autoencoder for Deep Learning of Images, Labels and Captions." Advances in 
542

543

544

545

546

547

548

549

550

551

552

553

554

555

556

557

558

559

560

561

562

563

564

565

566

567

568

569

570

571

572

573

574

575

576

577

578

579

580

Neural Information Processing Systems, September, 2352-60. http://arxiv.org/abs/1609.08976.

Rajaraman, Sivaramakrishnan, Sameer K Antani, Mahdieh Poostchi, Kamolrat Silamut, Md A Hossain, Richard J Maude, Stefan Jaeger, and George R Thoma. 2018. "Pre-Trained Convolutional Neural Networks as Feature Extractors toward Improved Malaria Parasite Detection in Thin Blood Smear Images." PeerJ 6 (April): e4568. https://doi.org/10.7717/peerj.4568.

Arora, Monika, and Vineet Kansal. 2019. "Character Level Embedding with Deep Convolutional Neural Network for Text Normalization of Unstructured Data for Twitter Sentiment Analysis." Social Network Analysis and Mining 9 (1): 12. https://doi.org/10.1007/s13278-019-0557-y.

Asgarian, Ehsan, Mohsen Kahani, and Shahla Sharifi. 2018. "The Impact of Sentiment Features on the Sentiment Polarity Classification in Persian Reviews." Cognitive Computation 10 (1): 117-35. https://doi.org/10.1007/s12559-017-9513-1.

Gan, Chenquan, Lu Wang, Zufan Zhang, and Zhangyi Wang. 2020. "Sparse Attention Based Separable Dilated Convolutional Neural Network for Targeted Sentiment Analysis." Knowledge-Based Systems 188 (January): 104827. https://doi.org/10.1016/j.knosys.2019.06.035.

Gers, F.A. 1999. "Learning to Forget: Continual Prediction with LSTM." In 9th International Conference on Artificial Neural Networks: ICANN '99, 1999:850-55. IEE. https://doi.org/10.1049/cp:19991218.

Graves, Alex, and Jürgen Schmidhuber. 2005. "Framewise Phoneme Classification with Bidirectional LSTM and Other Neural Network Architectures." Neural Networks 18 (5-6): 602-10. https://doi.org/10.1016/j.neunet.2005.06.042.

Greff, Klaus, Rupesh K. Srivastava, Jan Koutnik, Bas R. Steunebrink, and Jurgen Schmidhuber. 2017. "LSTM: A Search Space Odyssey." IEEE Transactions on Neural Networks and Learning Systems 28 (10): 2222-32. https://doi.org/10.1109/TNNLS.2016.2582924.

Hochreiter, Sepp, and Jürgen Schmidhuber. 1997. "Long Short-Term Memory." Neural Computation 9 (8): 1735-80. https://doi.org/10.1162/neco.1997.9.8.1735.

Kim, Yoon. 2014. "Convolutional Neural Networks for Sentence Classification." ArXiv Preprint ArXiv:1408.5882, August. http://arxiv.org/abs/1408.5882.

Kontopoulos, Efstratios, Christos Berberidis, Theologos Dergiades, and Nick Bassiliades. 2013. "Ontology-Based Sentiment Analysis of Twitter Posts." Expert Systems with Applications 40 (10): 4065-74. https://doi.org/10.1016/j.eswa.2013.01.001.

Montejo-Ráez, Arturo, Eugenio Martínez-Cámara, M. Teresa Martín-Valdivia, and L. Alfonso UreñaLópez. 2014. "Ranked WordNet Graph for Sentiment Polarity Classification in Twitter." Computer Speech \& Language 28 (1): 93-107. https://doi.org/10.1016/j.csl.2013.04.001.

Nguyen, Tuan-Linh, Swathi Kavuri, and Minho Lee. 2018. "A Fuzzy Convolutional Neural Network for Text Sentiment Analysis." Edited by Seong Oun Hwang. Journal of Intelligent \& Fuzzy Systems 35 (6): 6025-34. https://doi.org/10.3233/JIFS-169843.

Paltoglou, Georgios, and Mike Thelwall. 2012. "Twitter, MySpace, Digg." ACM Transactions on Intelligent Systems and Technology 3 (4): 1-19. https://doi.org/10.1145/2337542.2337551.

Rachum, Ram. 2020. "Regular Expression Operations." 2020. https://github.com/python/cpython/blob/3.9/Lib/re.py.

Peer) Comput. Sci. reviewing PDF | (CS-2020:10:54625:2:0:NEW 5 Feb 2021) 
581

582

583

584

585

586

587

588

589

590

591

592

593

594

595

596

597

598

599

600

601

602

603

604

605

606

607

608

609

610

611

612

613

614

615

616

617

618

619

620

621

Rao, Guozheng, Weihang Huang, Zhiyong Feng, and Qiong Cong. 2018. "LSTM with Sentence Representations for Document-Level Sentiment Classification." Neurocomputing 308 (September): 49-57. https://doi.org/10.1016/j.neucom.2018.04.045.

Richardson, Leonard. 2020. “Beautiful Soup 4.9.3." Crummy. 2020. https://www.crummy.com/software/BeautifulSoup/.

Schuster, M., and K.K. Paliwal. 1997. "Bidirectional Recurrent Neural Networks." IEEE Transactions on Signal Processing 45 (11): 2673-81. https://doi.org/10.1109/78.650093.

Vogels, W. 2016. "Bringing the Magic of Amazon Al and Alexa to Apps on AWS." 2016. https://www.allthingsdistributed.com/2016/11/amazon-ai-and-alexa-for-all-aws-apps.html.

Wehrmann, Joonatas, Willian Becker, Henry E. L. Cagnini, and Rodrigo C. Barros. 2017. "A CharacterBased Convolutional Neural Network for Language-Agnostic Twitter Sentiment Analysis." In 2017 International Joint Conference on Neural Networks (IJCNN), 2384-91. IEEE. https://doi.org/10.1109/IJCNN.2017.7966145.

Wilson, Theresa, Paul Hoffmann, Swapna Somasundaran, Jason Kessler, Janyce Wiebe, Yejin Choi, Claire Cardie, Ellen Riloff, and Siddharth Patwardhan. 2005. "OpinionFinder." In Proceedings of HLT/EMNLP on Interactive Demonstrations -, 34-35. Morristown, NJ, USA: Association for Computational Linguistics. https://doi.org/10.3115/1225733.1225751.

Wu, Yonghui, Mike Schuster, Zhifeng Chen, Quoc V. Le, Mohammad Norouzi, Wolfgang Macherey, Maxim Krikun, Yuan Cao, Qin Gao, Klaus Macherey, Jeff Klingner, Apurva Shah, Melvin Johnson, Xiaobing Liu, Łukasz Kaiser, Stephan Gouws, Yoshikiyo Kato, Taku Kudo, Hideto Kazawa, Keith Stevens, George Kurian, Nishant Patil, Wei Wang, Cliff Young, Jason Smith, Jason Riesa, Alex Rudnick, Oriol Vinyals, Greg Corrado, Macduff Hughes, Jeffrey Dean. 2016. "Google's Neural Machine Translation System: Bridging the Gap between Human and Machine Translation," September. http://arxiv.org/abs/1609.08144.

Yadav, Ashima, and Dinesh Kumar Vishwakarma. 2020. "Sentiment Analysis Using Deep Learning Architectures: A Review." Artificial Intelligence Review 53 (6): 4335-85. https://doi.org/10.1007/s10462-019-09794-5.

Ye, Qiang, Ziqiong Zhang, and Rob Law. 2009. "Sentiment Classification of Online Reviews to Travel Destinations by Supervised Machine Learning Approaches." Expert Systems with Applications 36 (3): 6527-35. https://doi.org/10.1016/j.eswa.2008.07.035.

Yildirim, Özal. 2018. "A Novel Wavelet Sequence Based on Deep Bidirectional LSTM Network Model for ECG Signal Classification." Computers in Biology and Medicine 96 (May): 189-202. https://doi.org/10.1016/j.compbiomed.2018.03.016.

Zhang, Ye, and Byron Wallace. 2015. "A Sensitivity Analysis of (and Practitioners' Guide to) Convolutional Neural Networks for Sentence Classification," October. http://arxiv.org/abs/1510.03820.

Roshanfekr, Behnam, Shahram Khadivi, and Mohammad Rahmati. 2017. "Sentiment Analysis Using Deep Learning on Persian Texts." In 2017 Iranian Conference on Electrical Engineering (ICEE), 1503-8. IEEE. https://doi.org/10.1109/IranianCEE.2017.7985281.

Saraee, Mohamad, and Ayoub Bagheri. 2013. "Feature Selection Methods in Persian Sentiment Analysis." In International Conference on Application of Natural Language to Information Systems, 303-8. Springer. https://doi.org/10.1007/978-3-642-38824-8_29.

Peer) Comput. Sci. reviewing PDF | (CS-2020:10:54625:2:0:NEW 5 Feb 2021) 
622

623

624

625

626

627

628

629

630

631

632

633

634

635

636

637

638

639

640

641

642

643

644

645

646

647

648

649

650

651

652

653

654

655

656

657

658

Severyn, Aliaksei, and Alessandro Moschitti. 2015. "Twitter Sentiment Analysis with Deep Convolutional Neural Networks." In Proceedings of the 38th International ACM SIGIR Conference on Research and Development in Information Retrieval - SIGIR '15, 959-62. New York, New York, USA: ACM Press. https://doi.org/10.1145/2766462.2767830.

Sokolova, Marina, Nathalie Japkowicz, and Stan Szpakowicz. 2006. "Beyond Accuracy, F-Score and ROC: A Family of Discriminant Measures for Performance Evaluation." In Australasian Joint Conference on Artificial Intelligence, 1015-21. Springer. https://doi.org/10.1007/11941439_114.

Taboada, Maite, Julian Brooke, Milan Tofiloski, Kimberly Voll, and Manfred Stede. 2011. "Lexicon-Based Methods for Sentiment Analysis." Computational Linguistics 37 (2): 267-307. https://doi.org/10.1162/COLI_a_00049.

Tang, Duyu, Bing Qin, and Ting Liu. 2015. "Deep Learning for Sentiment Analysis: Successful Approaches and Future Challenges." Wiley Interdisciplinary Reviews: Data Mining and Knowledge Discovery 5 (6): 292-303. https://doi.org/10.1002/widm.1171.

Tausczik, Yla R, and James W Pennebaker. 2010. "The Psychological Meaning of Words: LIWC and Computerized Text Analysis Methods." Journal of Language and Social Psychology 29 (1): 24-54. https://doi.org/10.1177/0261927X09351676.

Velupillai, Sumithra, Hercules Dalianis, Martin Hassel, and Gunnar H Nilsson. 2009. “Developing a Standard for De-Identifying Electronic Patient Records Written in Swedish: Precision, Recall and FMeasure in a Manual and Computerized Annotation Trial." International Journal of Medical Informatics 78 (12): e19-26. https://doi.org/10.1016/j.ijmedinf.2009.04.005.

Vorraboot, Piyanoot, Suwanna Rasmequan, Krisana Chinnasarn, and Chidchanok Lursinsap. 2015. "Improving Classification Rate Constrained to Imbalanced Data between Overlapped and NonOverlapped Regions by Hybrid Algorithms." Neurocomputing 152 (March): 429-43. https://doi.org/10.1016/j.neucom.2014.10.007.

Willett, Peter. 2006. "The Porter Stemming Algorithm: Then and Now." Program 40 (3): 219-23. https://doi.org/10.1108/00330330610681295.

Yazdinejad, Mohsen, and Sajjad Shumaly. 2020. "Persian Sentiment Analysis of an Online Store Using Convolutional Neural Network with FastText Embeddings." 2020. https://github.com/mosiomohsen/persian-sentiment-analysis-using-fastText-word-embeddingand-pseudo-labeling.

Zhang, Lei, Shuai Wang, and Bing Liu. 2018. "Deep Learning for Sentiment Analysis: A Survey." Wiley Interdisciplinary Reviews: Data Mining and Knowledge Discovery 8 (4): e1253. https://doi.org/10.1002/widm.1253.

Zhao, Hongshan, Huihai Liu, Wenjing Hu, and Xihui Yan. 2018. "Anomaly Detection and Fault Analysis of Wind Turbine Components Based on Deep Learning Network." Renewable Energy 127 (November): 825-34. https://doi.org/10.1016/j.renene.2018.05.024.

Peer] Comput. Sci. reviewing PDF | (CS-2020:10:54625:2:0:NEW 5 Feb 2021) 
Figure 1

A convolutional network architecture to sentiment classification

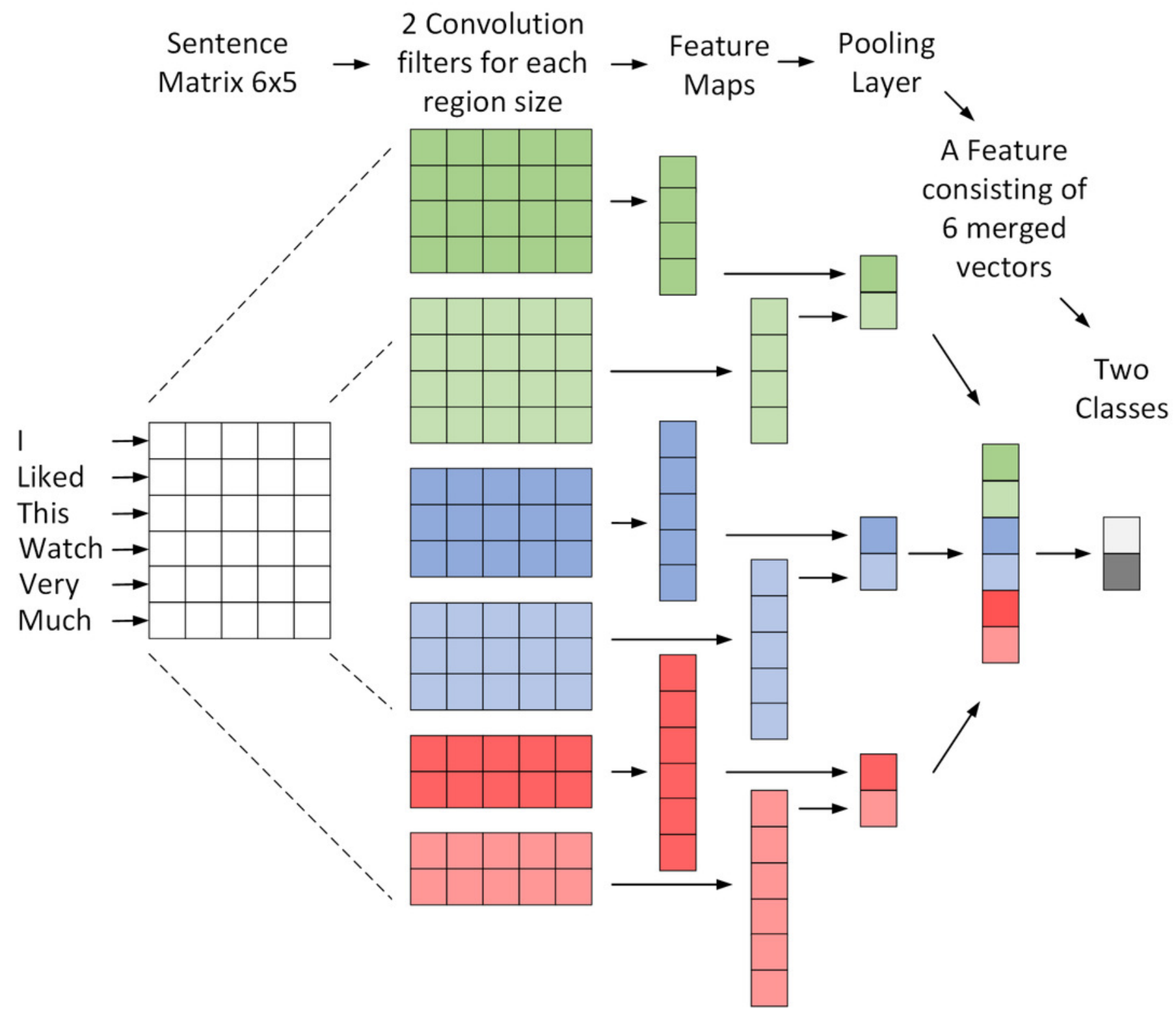


Figure 2

A demonstration of an LSTM cell

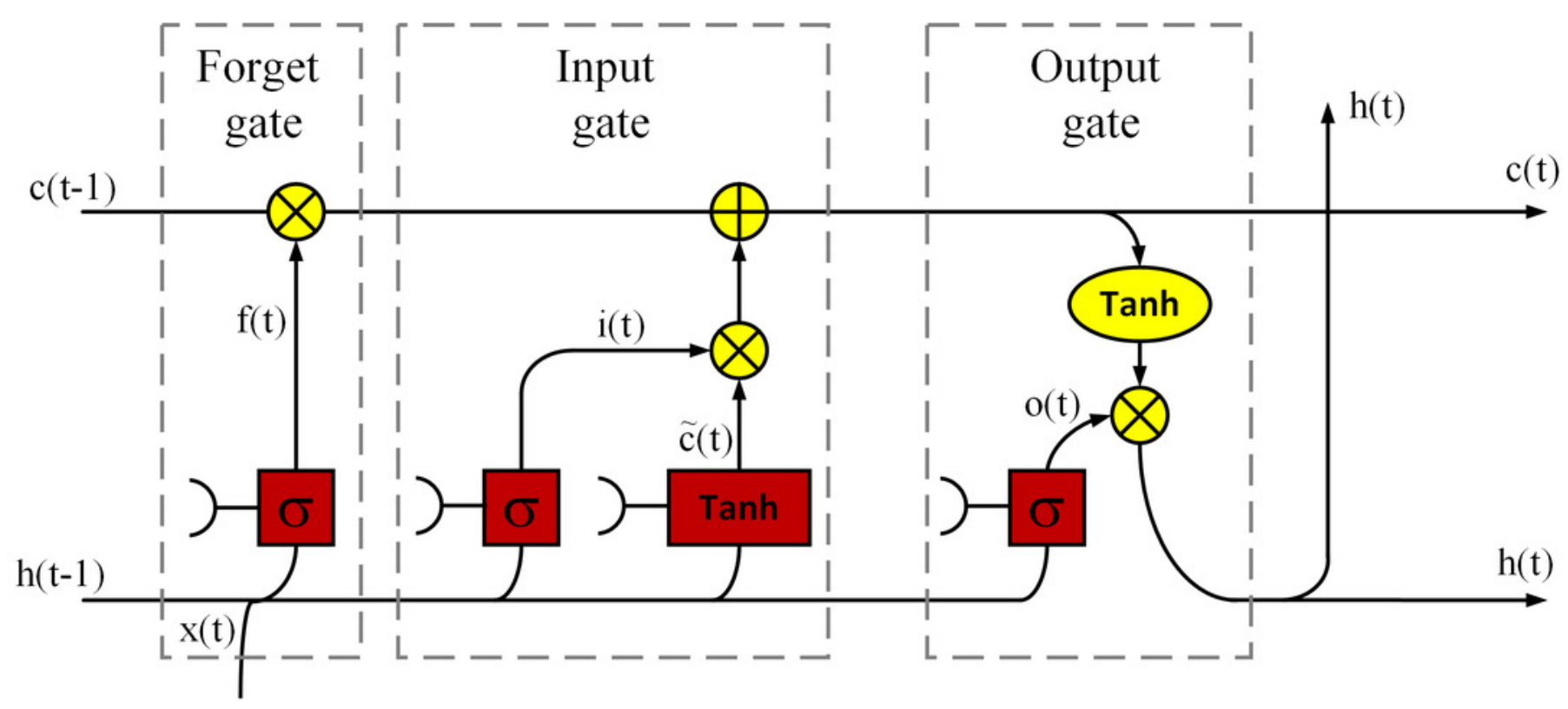


Figure 3

A basic structure of the BiLSTM network

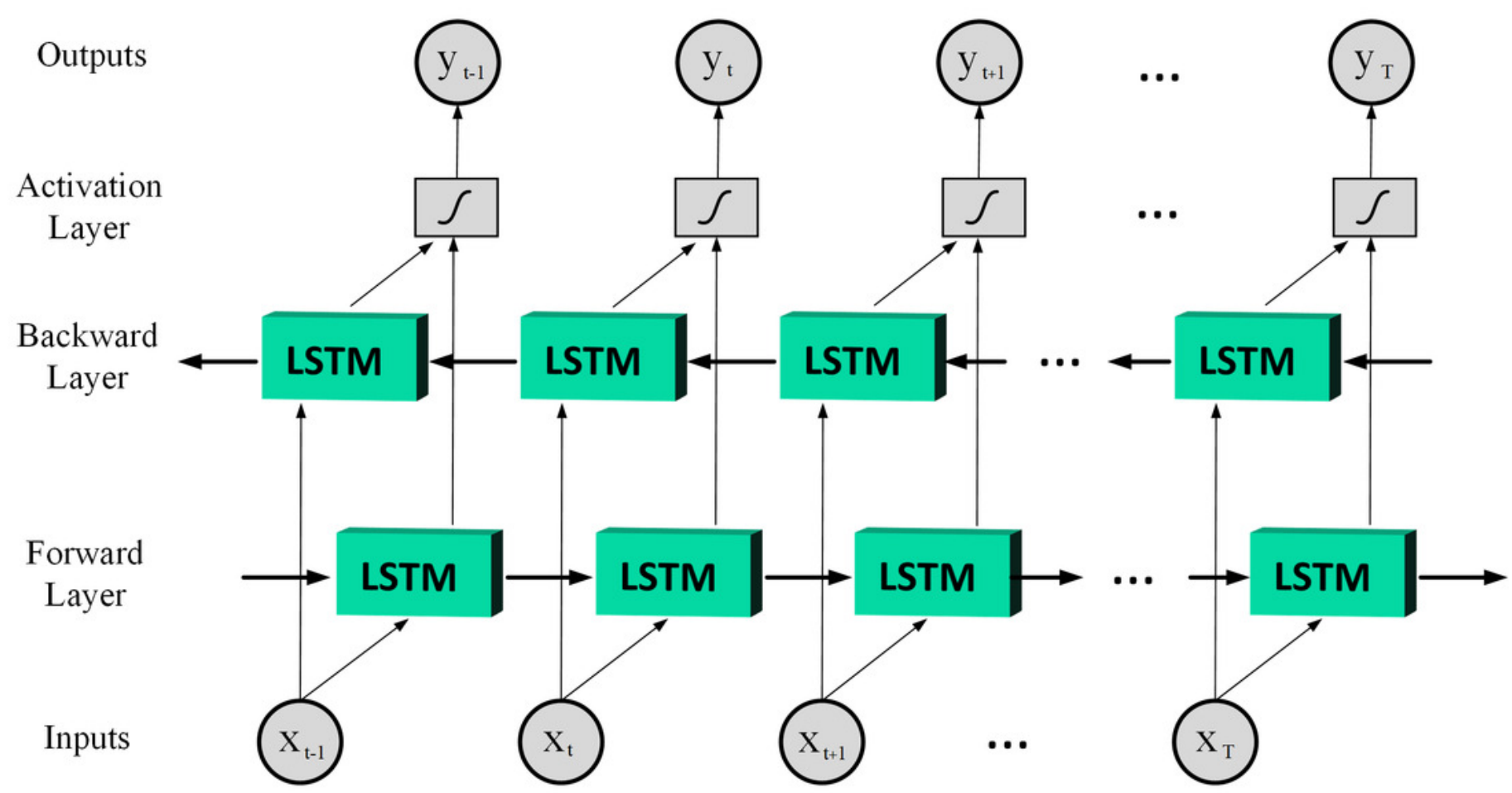


Figure 4

A flowchart representing the taken steps 


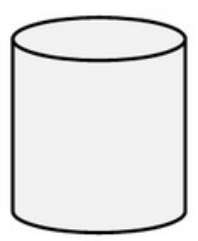

Online store website

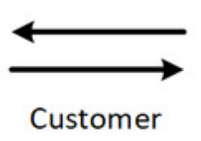

reviews about products

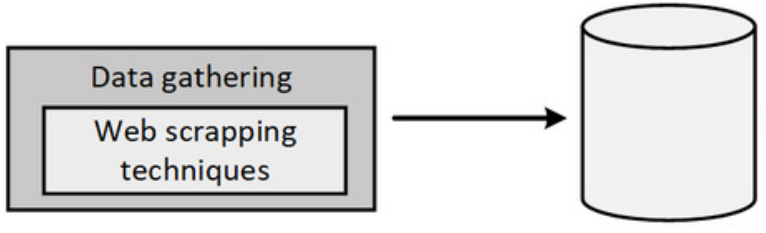

Unclean and imbalanced dataset
2,932,747 About $1.8 \mathrm{M}$ positive feedbacks reviews About $400 \mathrm{~K}$ negative feedbacks About $700 \mathrm{~K}$ is not specified

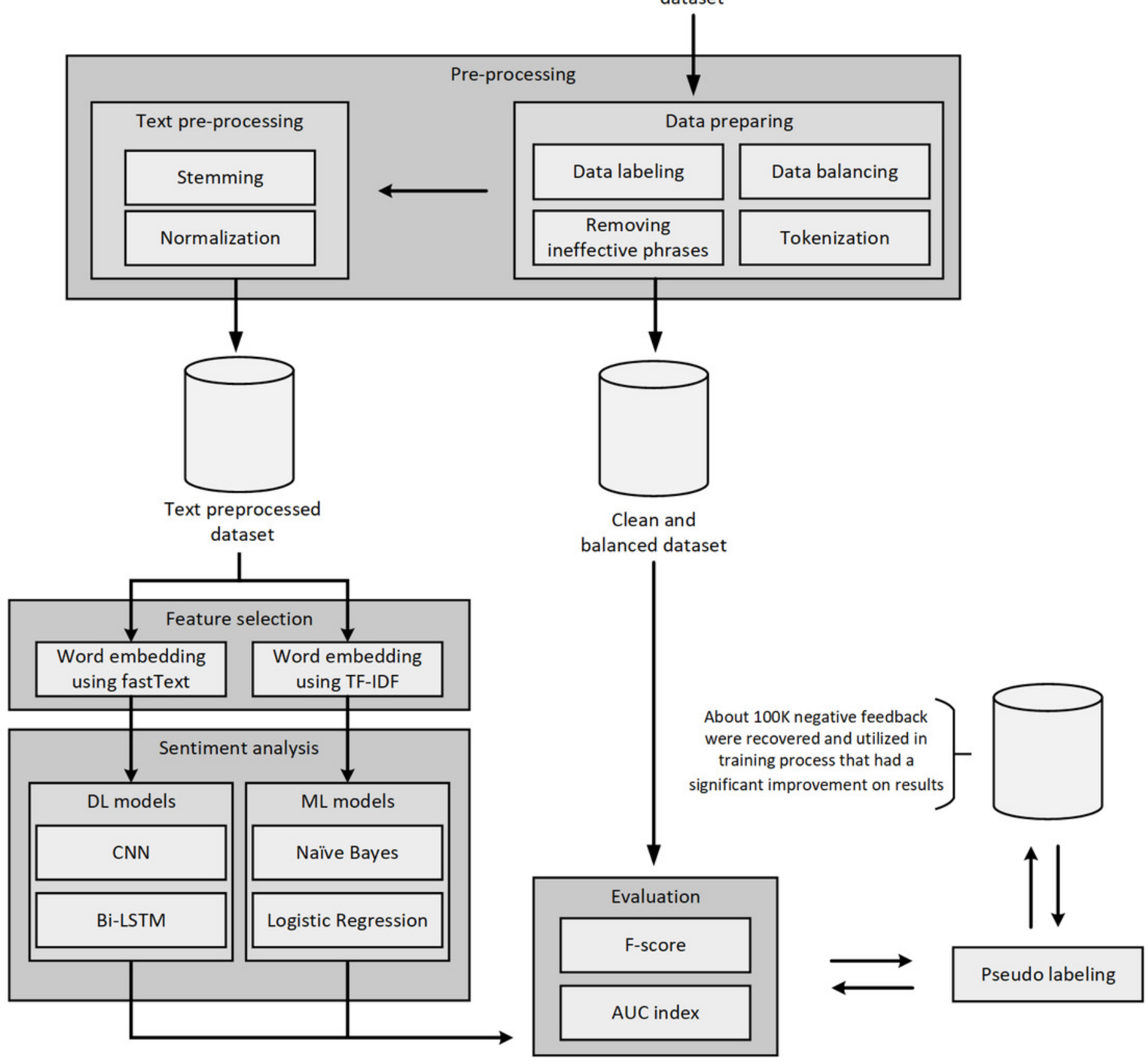


Figure 5

\section{Frequency of reviews per category}

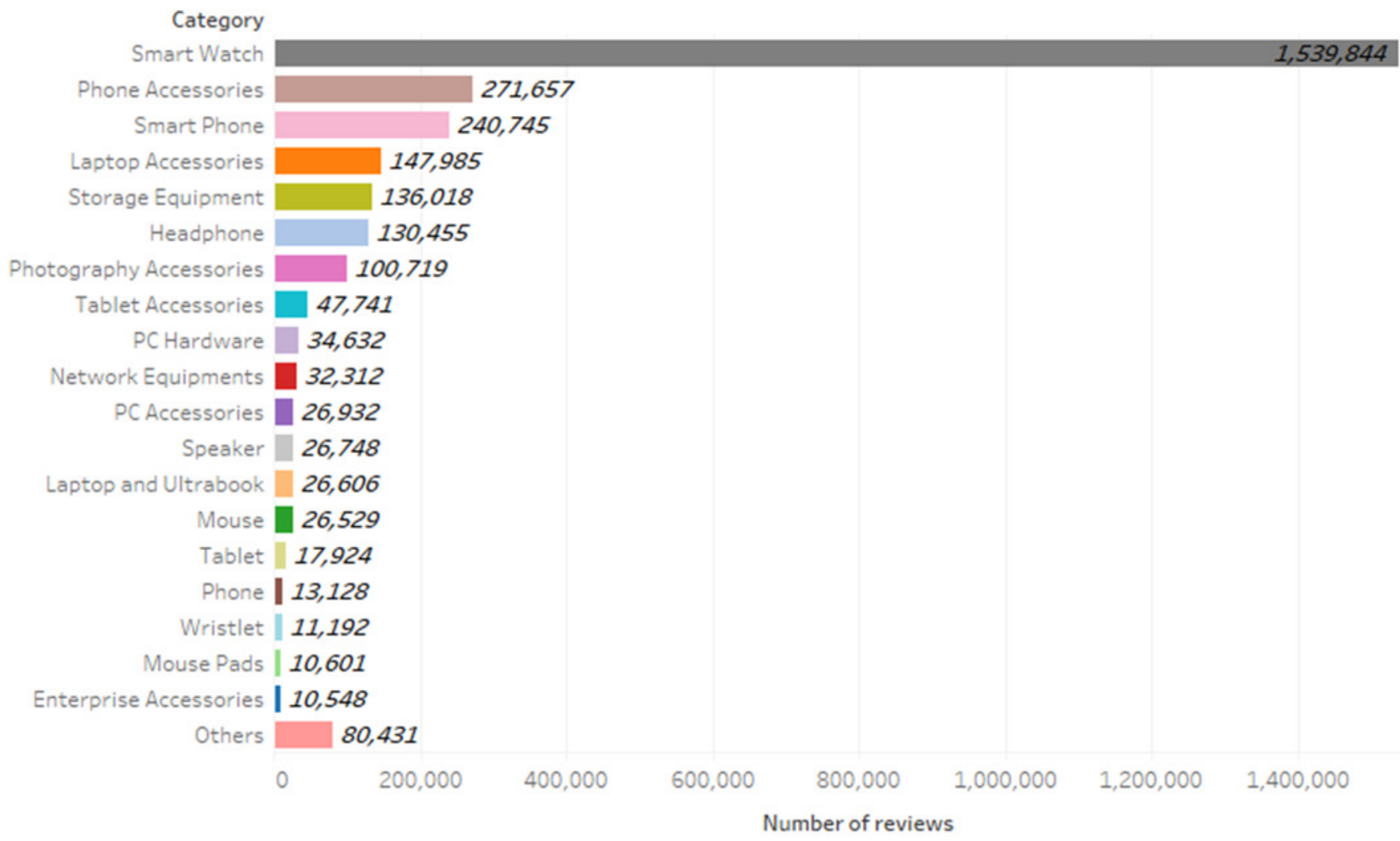




\section{Figure 6}

Positive feedback class word cloud

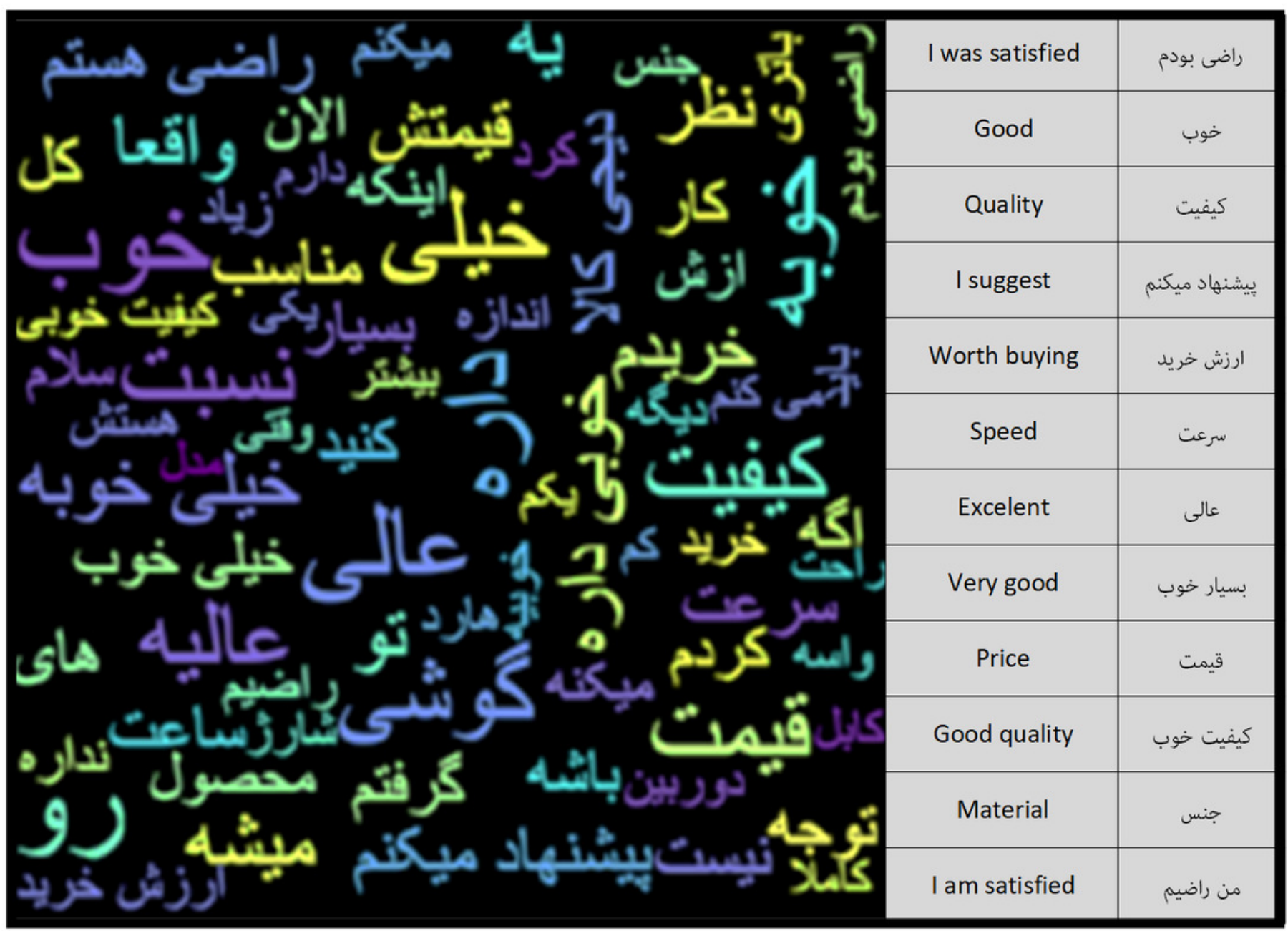




\section{Figure 7}

Negative feedback class word cloud

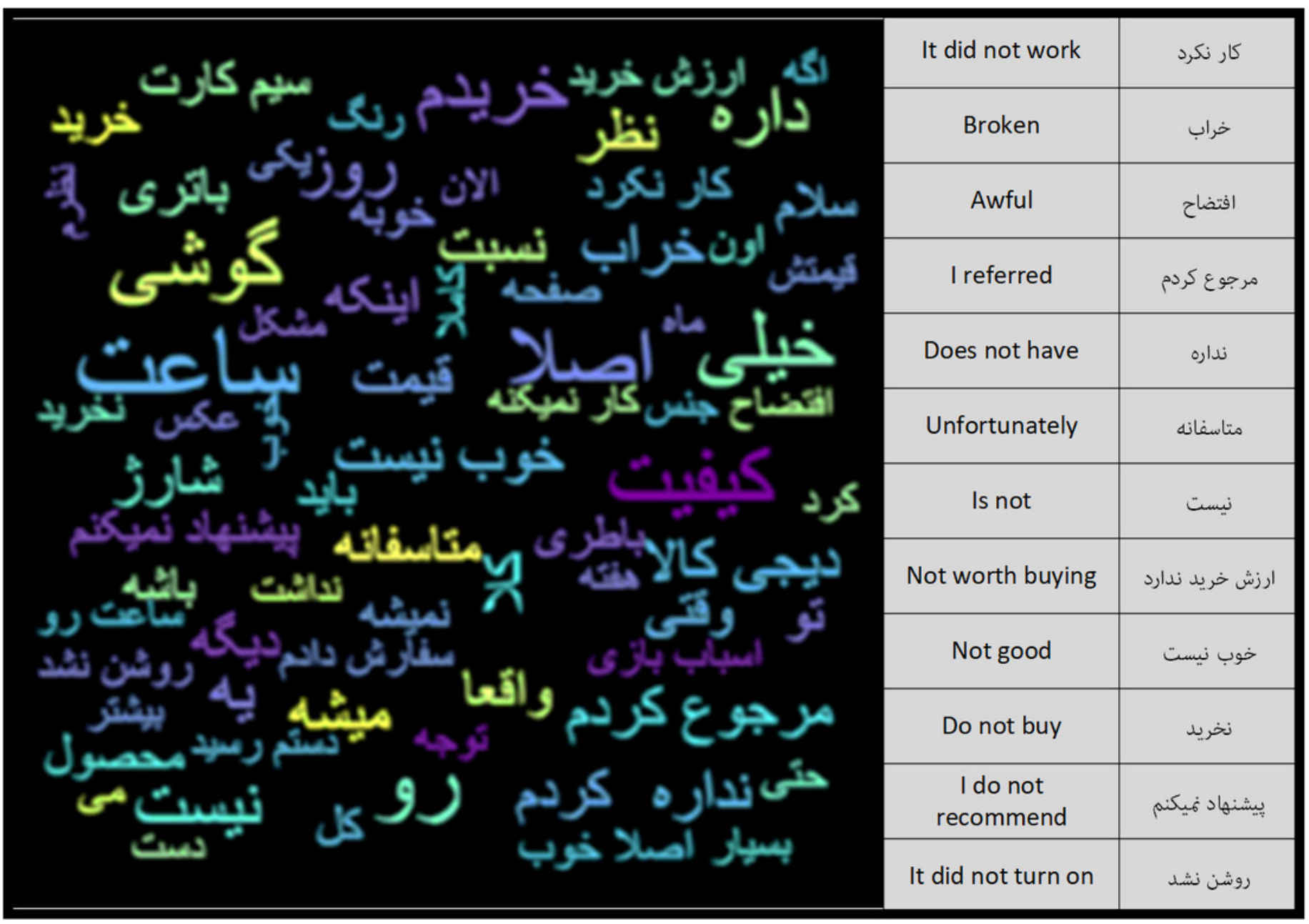


Figure 8

A: AUC before pre-processing ( $A U C=0.9943), B$ : AUC after pre-processing $(A \cup C=0.9944), C: A \cup C$ after pseudo labeling $(A \cup C=0.9996)$

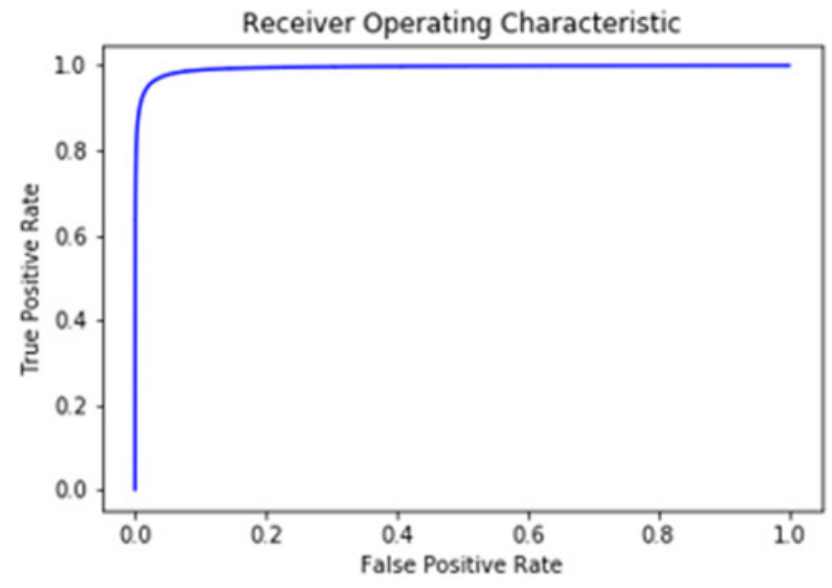

(A)

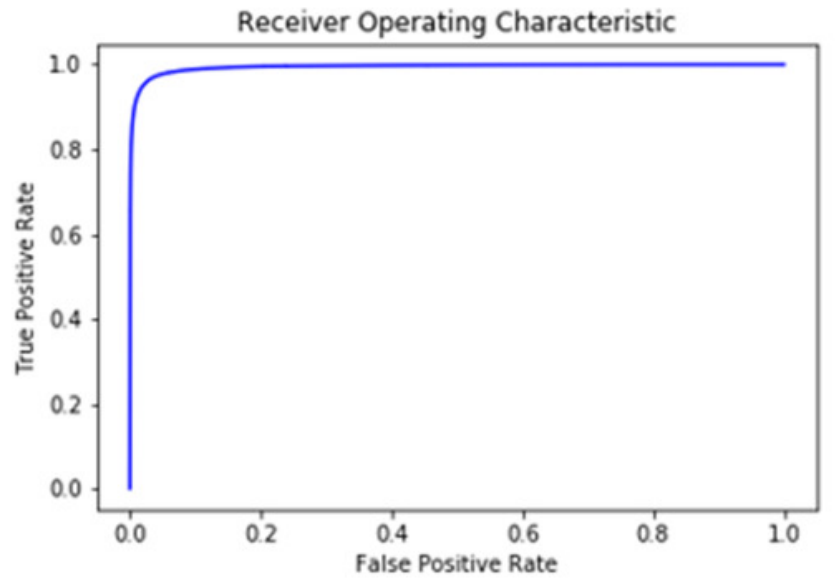

(B)

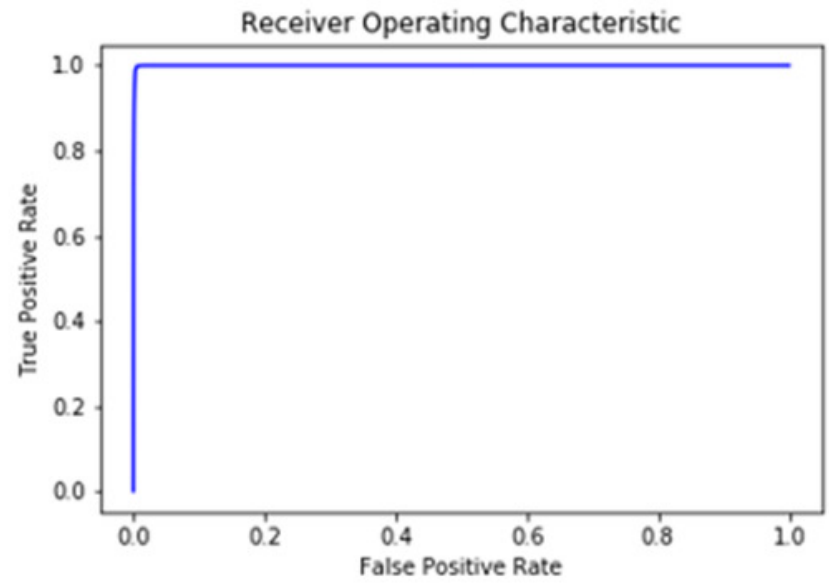

(C) 


\section{Table $\mathbf{1}$ (on next page)}

The CNN model structure 
Table 1 The CNN model structure

\begin{tabular}{|c|c|c|}
\hline Layer (type) & Output Shape & Number of Parameters \\
\hline embedding_2 (Embedding) & (None, 400, 100) & 11147700 \\
\hline dropout_8 (Dropout) & (None, 400, 100) & 0 \\
\hline conv1d (Conv1D) & (None, 400, 128) & 0 \\
\hline global_max_pooling1d (Global) & (None, 128) & 8256 \\
\hline dense_6 (Dense) & (None, 64) & 0 \\
\hline dropout_9 (Dropout) & (None, 64) & 0 \\
\hline dense_7 (Dense) & (None, 16) & 17 \\
\hline dropout_10 (Dropout) & (None, 16) & $11,195,541$ \\
\hline dense_8 (Dense) & (None, 1) & 0 \\
\hline \multicolumn{2}{|c|}{ Total parameters: } & \\
\hline
\end{tabular}

2 


\section{Table 2 (on next page)}

The BiLSTM model structure 
Table 2 The BiLSTM model structure

\begin{tabular}{|c|c|c|}
\hline Layer (type) & Output Shape & Number of Parameters \\
\hline embedding_1 (Embedding) & (None, 400, 100) & 11147700 \\
\hline dropout_4 (Dropout) & (None, 400, 100) & 0 \\
\hline bidirectional_1 (Bidirection) & (None, 64) & 0 \\
\hline dropout_5 (Dropout) & (None, 64) & 4160 \\
\hline dense_3 (Dense) & (None, 64) & 0 \\
\hline dropout_6 (Dropout) & (None, 64) & 0 \\
\hline dense_4 (Dense) & (None, 16) & 1040 \\
\hline dropout_7 (Dropout) & (None, 16) & $11,186,965$ \\
\hline dense_5 (Dense) & (None, 1) & 0 \\
\hline \multicolumn{2}{|c|}{ Total parameters: } & \\
\hline
\end{tabular}

2 


\title{
Table 3(on next page)
}

\author{
A sample of the collected dataset
}




\begin{tabular}{|c|c|c|c|c|c|}
\hline$\underset{\text { ID }}{\text { Comment }}$ & Original Comment & Translated Comment & $\begin{array}{l}\text { Negative } \\
\text { Feedback }\end{array}$ & $\begin{array}{l}\text { Positive } \\
\text { Feedback }\end{array}$ & Cat. Name \\
\hline 0 & 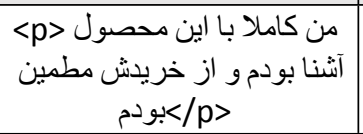 & $\begin{array}{l}\text { I was completely familiar } \\
\text { with this product and I } \\
\text { was sure of buying it }\end{array}$ & 0 & 1 & $\begin{array}{l}\text { Smart } \\
\text { Watch }\end{array}$ \\
\hline 31 & 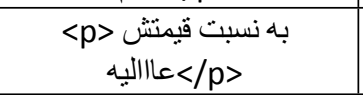 & Grrrreat for the price & 0 & 1 & $\begin{array}{l}\text { Smart } \\
\text { Watch }\end{array}$ \\
\hline 84278 & 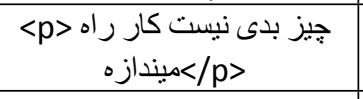 & Not a bad thing & 0 & 0 & $\begin{array}{c}\text { Phone } \\
\text { Accessories }\end{array}$ \\
\hline 3083 & 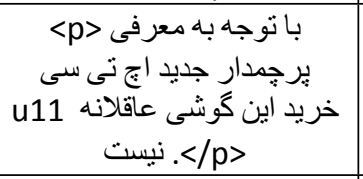 & $\begin{array}{c}\text { Given the introduction of } \\
\text { the new flagship HTC } \\
\text { u11, buying this phone is } \\
\text { not a wise choice }\end{array}$ & 1 & 0 & $\begin{array}{l}\text { Smart } \\
\text { Phone }\end{array}$ \\
\hline 1503094 & 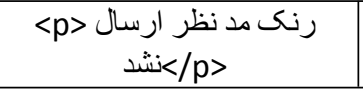 & $\begin{array}{c}\text { The requested color was } \\
\text { not sent }\end{array}$ & 1 & 0 & $\begin{array}{l}\text { Smart } \\
\text { Watch }\end{array}$ \\
\hline
\end{tabular}




\section{Table 4 (on next page)}

Performance of different models based on AUC and F-measure 


\begin{tabular}{|c|c|c|c|c|c|c|c|c|}
\hline States & Index & Fold1 & Fold2 & Fold 3 & Fold 4 & Fold 5 & Mean & Error (SEM) \\
\hline \multirow{2}{*}{ BiLSTM } & AUC: & 0.9934 & 0.9937 & 0.993 & 0.993 & 0.9934 & 0.9933 & $13.4 * 10^{-5}$ \\
\cline { 2 - 9 } & F-score: & 0.9224 & 0.9244 & 0.9238 & 0.9216 & 0.9232 & 0.9230 & $49.6 * 10^{-5}$ \\
\hline \multirow{2}{*}{ CNN } & AUC: & 0.9945 & 0.9945 & 0.9943 & 0.9946 & 0.9945 & 0.9944 & $4.9 * 10^{-5}$ \\
\cline { 2 - 9 } & F-score: & 0.9293 & 0.9251 & 0.9306 & 0.9299 & 0.93 & 0.9289 & $99.2 * 10^{-5}$ \\
\hline \multirow{2}{*}{$\begin{array}{c}\text { Naïve } \\
\text { Bayes }\end{array}$} & AUC: & 0.9877 & 0.9881 & 0.9878 & 0.988 & 0.9881 & 0.9879 & $8.12^{*} 10^{-5}$ \\
\cline { 2 - 9 } & F-score: & 0.8856 & 0.8856 & 0.886 & 0.8863 & 0.8863 & 0.8859 & $15.7 * 10^{-5}$ \\
\hline \multirow{2}{*}{$\begin{array}{c}\text { Logistic } \\
\text { Regression }\end{array}$} & AUC: & 0.9888 & 0.9891 & 0.9888 & 0.989 & 0.9881 & 0.9887 & $17.5^{*} 10^{-5}$ \\
\cline { 2 - 9 } & F-score: & 0.8894 & 0.8901 & 0.8898 & 0.8895 & 0.8863 & 0.8890 & $69.1 * 10^{-5}$ \\
\hline
\end{tabular}

2 


\section{Table 5 (on next page)}

Performance of the CNN model in different situations based on AUC and F-measure 


\begin{tabular}{|c|c|c|c|c|c|c|c|c|}
\hline States & Index & Fold1 & Fold2 & Fold 3 & Fold 4 & Fold 5 & Mean & Error (SEM) \\
\hline \multirow{2}{*}{ Before Prep. } & AUC: & 0.9943 & 0.9943 & 0.9944 & 0.9944 & 0.9945 & 0.994 & $3.7 * 10^{-5}$ \\
\cline { 2 - 9 } & F-score: & 0.928 & 0.9298 & 0.9303 & 0.9271 & 0.9304 & 0.929 & $66.4^{*} 10^{-5}$ \\
\hline \multirow{2}{*}{ After Prep. } & AUC: & 0.9945 & 0.9945 & 0.9943 & 0.9946 & 0.9945 & 0.994 & $4.8^{*} 10^{-5}$ \\
\cline { 2 - 9 } & F-score: & 0.9293 & 0.9251 & 0.9306 & 0.9299 & 0.93 & 0.928 & $99.1^{*} 10^{-5}$ \\
\hline $\begin{array}{c}\text { After Pseudo } \\
\text { labeling }\end{array}$ & AUC: & 0.9944 & 0.9943 & 0.9946 & 0.9995 & 0.9996 & 0.996 & $12.5^{*} 10^{-5}$ \\
\cline { 2 - 9 } & F-score: & 0.9431 & 0.9434 & 0.9443 & 0.9767 & 0.9758 & 0.956 & $80^{*} 10^{-5}$ \\
\hline
\end{tabular}

2 